VOL. $31(1985), 171-179$.

\title{
ON THE FOURIER TRANSFORMS
}

\author{
HWAI-CHIUAN WANG
}

\begin{abstract}
In this article we give a new proof of the theorem that a positive even convex function on the real line, which vanishes at infinity, is the Fourier transform of an integrable function. Related results in several variables are also proved. As an application of our results we solve the factorization problem of Sobolev algebras.
\end{abstract}

\section{0 . Introduction}

Recall several classical Fourier multiplier results: Katznelson [2, p. 22] proved that a positive even convex sequence vanishing at infinity is the sequence of Fourier coefficients of an integrable function. Titchmarch $[5$, p. 170] proved that a positive even convex function on the real line vanishing at infinity is the Fourier cosine transform of an integrable function. However, [5] asserted that there exists a positive even convex function on the real line vanishing at infinity which is not the Fourier sine transform of an integrable function. In section 1 we prove that a positive even convex function on the real line vanishing at infinity is the Fourier transform of an integrable function (see Theorem 1.1). This is not a new result but we use a simple and delicate method through the GaussWeierstrass kernels, which is closely related to partial differential

Received 21 September 1984. This research is supported in part by the National Science Council, Republic of China. The author would like to express his sincere gratitude to Professor R.R. Goldberg for his lectures drawing the author's attention to this problem.

Copyright Clearance Centre, Inc. Serial-fee code: 0004-9727/85 $\$ A 2.00+0.00$. 
equations. We hope in the future to apply our present results to some problems in partial differential equations. The same theory in several variables is not clear yet. We also obtain partial results in the case of several variables. In Section 2 we apply the theorems of Section 1 to solve the factorization problem of Sobolev algebras on the real line.

\section{Main results}

Throughout this article we denote by $R^{n}$ the $n$-dimensional euclidean space.

THEOREM 1.1. Let $f(x)$ be an even positive convex fronction vanishing at infinity, then there is a positive function $F$ in $L^{1}(R)$ such that $f$ is the Eourier transform of $F$.

Proof. We divide the proof into two steps.

(1) From the hypothesis the derivative $f^{\prime}$ of $f$ is nonpositive, nondecreasing and

$$
f(x)=\int_{-\infty}^{x} f^{\prime}(t) d t .
$$

For nonzero real $u$,

$$
\begin{aligned}
\int_{R} f(x) e^{2 \pi i u x} d x & =2 \int_{0}^{\infty} f(x) \cos 2 \pi u x d x \\
& =-1 / \pi|u| \int_{0}^{\infty} f^{\prime}(x) \sin 2 \pi|u| x d x \\
& =1 / \pi|u| \sum_{j=0}^{\infty} \int_{j / 2|u|}^{(j+1) / 2|u|}\left(-f^{\prime}(x) \sin 2 \pi|u| x\right) d x
\end{aligned}
$$

The series in the last equality is an alternating series with positive first term, nonincreasing in absolute values of terms, and vanishing at infinity. Therefore

$$
\int_{R} f(x) e^{2 \pi i u x} d x \geq 0
$$

(2) If we fix $U, S, 0<U \leq|u| \leq S$, then 


$$
\begin{aligned}
\left|\int_{T^{\prime} \geq|x| \geq T>0} f(x) e^{2 \pi i u x} d x\right| & =\left|\int_{T^{\prime} \geq|x| \geq T>0} f(x) \cos 2 \pi u x d x\right| \\
& =\left|f(c) \int_{T^{\prime} \geq|x| \geq T>0} \cos 2 \pi u x d x\right| \\
& \leq f(T) / \pi U \rightarrow 0 \text { as } T \rightarrow \infty .
\end{aligned}
$$

Using the above uniform convergence arguments, we obtain

$$
\begin{aligned}
& \int_{R}\left(\int_{R} f(x) e^{2 \pi i u x} d x\right) e^{-4 \pi^{2} a|u|^{2}} d u \\
& =\lim _{\substack{U \rightarrow 0 \\
S \rightarrow \infty}} \int_{0<U \leq|u| \leq S}\left(\int_{R} f(x) e^{2 \pi i u x} d x\right) e^{-4 \pi^{2} a|u|^{2}} d u \\
& =\lim _{\substack{U \rightarrow 0 \\
S \rightarrow \infty}} \lim _{T \rightarrow \infty} \int_{0<U \leq|u| \leq S}\left(\int_{|x| \leq T} f(x) e^{2 \pi i u x} d x\right) e^{-4 \pi^{2} a|u|^{2}} d u \\
& =\lim _{\substack{U \rightarrow \infty \\
S \rightarrow \infty}} \int_{R}\left(\int_{0<|U| \leq|u| \leq S} e^{-4 \pi^{2} a|u|^{2}} e^{2 \pi i u x} d u\right) f(x) d x \\
& =\int_{R}\left(\int_{R} e^{-4 \pi^{2} a|u|^{2}} e^{2 \pi i u x} d u\right) f(x) d x \\
& =\int_{R} f(x)(4 \pi a)^{-1 / 2} e^{-|x|^{2} / 4 a} d x \text {. }
\end{aligned}
$$

Since the Gauss-Weierstrass kernel $\left\{(4 \pi a)^{-1 / 2} e^{-|x|^{2} / 4 a}\right\} a>0$ is an approximation identity, we have

$$
\lim _{a \rightarrow 0} \int_{R} f(x)(4 \pi a)^{-1 / 2} e^{-x^{2} / 4 a} d x=f(0) .
$$

Thus

$$
\lim _{a \rightarrow 0} \int_{R}\left(\int_{R} f(x) e^{2 \pi i u x} d x\right) e^{-4 \pi^{2} a|u|^{2}} d u=f(0)
$$

However, from part (1),

$$
\int_{R} f(x) e^{2 \pi i u x} d x \geq 0 \text { for all real } u \text {. }
$$


By the Fatou lemma,

$$
\int_{R}\left(\int_{R} f(x) e^{2 \pi i u x} d x\right) d u \leq f(0)<\infty .
$$

Let

$$
F(u)=\int_{R} f(x) e^{2 \pi i u x} d x
$$

Then $F$ is positive integrable on the real line and

$$
\hat{F}=f \text {. }
$$

This completes the proof.

REMARK 1.2. Let $g(x)$ be a function on the real line vanishing at infinity. We take a positive even convex function $f(x)$ on the real line vanishing at infinity with $|g(x)| \leq f(x)$. Then by Theorem 1.1 , there is an integrable function $F$ such that $\hat{F}=f$. This property reveals that the Fourier transform of an integrable function on the real line can vanish at infinity arbitrarily slowly.

REMARK 1.3. By Theorem 1.1, a positive even convex function on the real line vanishing at infinity is a Fourier multiplier of p-integrable functions, $1 \leq p \leq \infty$.

REMARK 1.4. Theorem 1.1 may fail if in the hypothes is we replace even by odd. Let

$$
g(x)=\left\{\begin{array}{l}
1 / \log x \text { for } x>5, \\
\operatorname{tangent} \operatorname{line} \text { of } 1 / \log x \text { at } x=5 \text { for } 0 \leq x \leq 5, \\
-g(-x) \text { for } x<0,
\end{array}\right.
$$

then $g$ is not the Fourier transform of an integrable function (see Stein and Weiss $[4, p .31])$.

We consider below some related results in several variables.

PROPOSITION 1.5. Let $f(x)$ be a locally integrable function on $k^{n}$, which vanishes at infinity. If there is a positive even integer $N>n$ such that

$$
\partial_{x_{i}}^{N} f(x) \in L^{1}\left(R^{n}\right) \text { for } i=1,2, \ldots, n,
$$


and the inverse Fourier transform $F$ of $f$ is locally integrable, then $F \in L^{l}\left(R^{n}\right)$ with $\hat{F}=f$.

Proof. By the hypothesis

$$
\left(\partial_{x_{1}}^{N}+\ldots+\partial_{x_{n}}^{N}\right) f(x) \quad L^{1}\left(R^{n}\right)
$$

Let $F=\check{f} ;$ then

$$
(2 \pi i)^{N}\left(x_{1}^{N}+\ldots+x_{n}^{N}\right) F(x)
$$

is bounded for every $x \in R^{n}$. But

$$
\begin{aligned}
\left|x_{1}^{N}+\ldots+x_{n}^{N}\right| & \geq c\left[\left(x_{1}^{2}\right)^{N / 2}+\left(x_{n}^{2}\right)^{N / 2}\right] \\
& \geq c\left(x_{1}^{2}+\ldots+x_{n}^{2}\right)^{N / 2} .
\end{aligned}
$$

So

$$
|F(x)| \leq c /|x|^{N} \text { for large } x \text {. }
$$

Thus $F \in L^{l}\left(R^{n}\right)$ and $\hat{F}=f$.

EXAMPLE 1.6. Let $f(x)$ be a function on $k^{n}$ with

$$
f(x)=1 /|x|^{a} \text { for } x \neq 0,0<a<n,
$$

then it is known that the inverse Fourier transform $F$ of $f$ is of the form $c /|x|^{n-a}$, which is locally integrable. Also

$$
\left|\partial_{x_{i}}^{2 n} f(x)\right| \leq c /|x|^{a+2 n}, i=1,2, \ldots, n \text {. }
$$

So, by Proposition 1.5, $F \in L^{1}\left(R^{n}\right)$ with $\hat{F}=f$.

The following locally integrable characterization is useful, in view of Theorem 1.1.

PROPOSITION 1.7. Let $f(x)$ be a radial function on $R^{n}$, vanishing at infinity, $n>2$. Suppose $|\operatorname{grad} f(x)| \in L^{1}\left(R^{n}\right)$; then the inverse Fourier transform of $f$ is a locally integrable froction. 
Proof. Recall that

$$
\operatorname{lgrad} f(x)|=-\partial f(x) / \partial| x\left|=-f^{\prime}(x), \quad r=\right| x \mid \text {. }
$$

Using the polar representation, we have

$$
\omega_{n-1} \int_{0}^{\infty} r^{n-1}\left|f^{\prime}(r)\right| d r=\int_{R^{n}}|\operatorname{grad} f(x)| d x<\infty
$$

where $\omega_{n-1}$ is the area of the unit sphere in $k^{n}$. But

$$
f(r)=-\int_{r}^{\infty} f^{\prime}(t) d t
$$

or

$$
\begin{aligned}
|f(r)| & \leq \int_{r}^{\infty}\left|f^{\prime}(t)\right| d t \\
& \leq 1 / r^{n-1} \int_{0}^{\infty} t^{n-1}\left|f^{\prime}(t)\right| d t \\
& \leq c / r^{n-1} .
\end{aligned}
$$

Thus $f$ is locally integrable and

$$
\int_{1 \leq|x|}|f(x)|^{2} d x \leq c w_{n-1} \int_{1}^{\infty} 1 / r^{n-1} d x<\infty \text { if } n>2 \text {. }
$$

Let

$$
\begin{aligned}
f & =X_{\{x:|x| \leq I\}} f+X_{\{x:|x|>I\}} f \\
& =f_{1}+f_{2} .
\end{aligned}
$$

Then $f_{1} \in L^{1}\left(R^{n}\right), f_{2} \in L^{2}\left(R^{n}\right)$ with $\check{f}=\check{f}_{1}+\check{f}_{2}$. Thus $\bar{f}$ is locally integrable.

\section{Application}

We begin with several definitions.

DEFINITION 2.1. A Banach algebra $B$ is said to admit weak factorization if, given $h \in B$, there are $f_{1}, \ldots, f_{n}, g_{1}, \ldots, g_{n} \in B$ 
such that $h=\sum_{i=1}^{n} f_{i} g_{i}$.

DEFINITION 2.2. Let $\left(B(R),\|\|_{B}\right)$ be a Banach algebra of complexvalued measurable functions on $R . B(R)$ is called a homogeneous Banach algebra if it satisfies the following properties:

H1. if $f \in B(R), x \in R$ then $L_{x} f \in B(R),\left\|L_{x} f\right\|_{B}=\|f\|_{B}$ where

$$
L_{x} f(y)=f(y-x) \text { for } y \in R
$$

H2. $x \rightarrow L_{x} f$ is continuous from $R$ into $\left(B(R),\|\|_{R}\right)$.

The homogeneous Banach algebra $\left(B(R),\|\|_{R}\right)$ is a Segal algebra if $B(R)$ is dense in $L^{\perp}(R)$. A Segal algebra $B(R)$ is character invariant if $e^{i x t} f(t) \in B(R)$ whenever $x \in R, f \in B(R)$. The definitions and theory of Segal algebras can be established on general locally compact abelian groups instead of the real group $R$ (see Reiter [3] and Wang [6]).

DEFINITION 2.3. Let $H_{S}(R)$ be the space of all integrable functions on the real line such that

$$
\int_{R}\left(1+|x|^{2}\right)^{s}|\hat{u}(x)|^{2} d x<\infty .
$$

Let $\Lambda_{S}=\left(I-(2 \pi)^{-2} \Delta\right)^{s / 2}$ where $\Delta$ is the Laplacian, or

$$
\left(\widehat{\Lambda_{s} u}\right)(x)=\left(1+|x|^{2}\right)^{s / 2} \hat{u}(x) \text { for } x \in R
$$

define

$$
\|u\|_{H_{s}}=\|u\|_{L^{1}}+\left\|\Lambda_{s} u\right\|_{L^{2}}
$$

Using the techniques developed in Wang [7], we have

PROPOSITION 2.4. For any real $s,\left(H_{s}(R),\|\|_{H_{s}}\right)$ is a character invariant Segal algebra on $R$.

We then call $H_{S}(R)$ a Sobolev algebra. These are interesting and 
useful in both analysis and partial differential equations.

Furthermore we have the following factorization results.

THEOREM 2.5. The Sobolev algebra $H_{s}(R)$ admits a weak factorization if and only if $s<-1 / 2$.

Proof. (1) For $s<-1 / 2$ and $u \in L^{1}(R)$, then

$$
\begin{aligned}
\left\|\left(1+|x|^{2}\right)^{s / 2} \hat{u}(x)\right\|_{L^{2}}^{2} & =\int_{R}\left(1+|x|^{2}\right)^{s}|\hat{u}(x)|^{2} d x \\
& <\infty \text { since } \hat{u} \in C_{0}(R) .
\end{aligned}
$$

Thus $H_{s}(R)=L^{l}(R)$ for $s<-l / 2$. By a well-known factorization theorem of Cohen, the algebras $H_{s}(R), s<-l / 2$, admit weak factorizations.

(2) Feichtinger, Graham and Lakien [1] asserted that a character invariant Segal algebra $B(G)$ on a locally compact abelian group $G$ admits weak factorization if and only if $B(G)=L^{1}(G)$. Hence in order to prove that the algebras $H_{s}(R), s \geq-1 / 2$, do not admit weak

factorizations, it suffices to prove that $H_{s}(R) \varsubsetneqq L^{1}(R)$. However it is easy to see that the algebras $H_{s}(R)$ possess the going down property, $H_{s}(R) \subset H_{t}(R)$ if $s \geq t$. Thus it suffices to prove

$$
H_{-1 / 2}(R) \varsubsetneqq L^{1}(R)
$$

Let $f(x)$ be an even function on $R$ defined by

$$
f(x)=\left\{\begin{array}{l}
(\log x)^{-1 / 2} \text { for } x \geq 2, \\
\left(-1 / 4(\log 2)^{-3 / 2}\right)(x-2)+(\log 2)^{-1 / 2} \text { for } 0 \leq x<2 .
\end{array}\right.
$$

Actually the function $\left(-1 / 4(\log 2)^{-3 / 2}\right)(x-2)+(\log 2)^{-1 / 2}$ is the tangent line of the function $(\log x)^{-1 / 2}$ at $x=2$. Then $f$ is an even positive convex function on $R$ vanishing at infinity. By Theorem 1.1, there is an integrable function $u$ on $R$ such that $\hat{u}=f$. But 


$$
\begin{aligned}
\int_{R}\left(1+|x|^{2}\right)^{-1 / 2}|\hat{u}(x)|^{2} d x & \geq 2 \int_{2}^{\infty} \frac{1}{\left(1+x^{2}\right)^{\frac{1}{2}} \log x} d x \\
& \geq \int_{2} \frac{1}{x \log x} d x=\infty
\end{aligned}
$$

Thus $H_{-1 / 2}(R) \varsubsetneqq L^{1}(R)$, which completes the proof.

\section{References}

[1] Hans G. Feichtinger, Colin C. Graham and Eric H. Lakien, "Nonfactorization in commutative, weakly self-adjoint Banach algebras", Pacific J. Math. 80 (1979), 117-125.

[2] Y. Katznelson, An introduction to harmonic analysis (John Wiley \& Sons, New York, London, 1968).

[3] Hans Reiter, Classical harmonic analysis and locally compact groups (Clarendon Press, oxford, 1968).

[4] Elias M. Stein and Guido Weiss, Introduction to Fourier analysis on Euclidean spaces (Princeton Mathematical Series, 32. Princeton University :Press, Princeton, New Jersey, 1971).

[5] E.C. Titchmarch, Introduction to the theory of Fourier integrals (Clarendon Press, Oxford, 1959).

[6] Hwai-Chiuan Wang, Homogeneous Banach algebras (Lecture Notes in Pure and Applied Mathematics, 29. Marcel Dekker, New York, Basel, 1977).

[7] Hwai-chiuan Wang, "A note on Segal algebras on Euclidean spaces", Proc. Amer. Math. Soc. 72 (1978), 513-518.

Department of Mathematics,

National Tsing Hua University,

Hsinchu,

Taiwan 300,

Republic of China. 